\title{
Expression profile analysis of long non- coding RNA in skeletal muscle of osteoporosis by microarray and bioinformatics
}

\author{
Shaojin Liu', Hongxing Huang ${ }^{2}$, Shuang Chai ${ }^{1}$, Hewei Wei ${ }^{2}$, Jiachun Huang ${ }^{1}$ and Lei Wan ${ }^{2,3^{*}}$ (D)
}

\begin{abstract}
Background: Osteoporosis (OP) is a condition featured by bone mass loss and bone tissue microarchitectural alterations due to impaired tissue homeostasis favoring excessive bone resorption versus deposition. The trigger of such an impairment and the downstream molecular pathways involved are yet to be clarified. Long non-coding RNA (IncRNA) plays a role in gene transcription, protein expression and epigenetic regulation; and altered expression results in immune or metabolism related desease development. To determine whether IncRNAs are involved in osteoporosis, we analyzed the expression profile of IncRNAs and mRNAs in osteoporosis.
\end{abstract}

Method: Three pairs of osteoporosis patients (OP group) and healthy people controls (NC group) were screened by microarray. Quantitative polymerase chain reaction (qRT-PCR) was performed to confirm dysregulated IncRNA expressions in 5 pairs of OP and NC group tissues samples. Gene Ontology (GO) and Kyoto Encyclopedia of Genes and Genomes (KEGG) pathway analyses were performed to construct the IncRNA-mRNA co-expression network.

Result: Through co-expression analysis, differently expressed transcripts were divided into modules, and IncRNAs were functionally annotated. We further analyzed the clinical significance of crucial IncRNAs from modules in public data. Finally, the expression of five IncRNAs, CUST_44695_PI430048170-GeneSymbol:CTA-384D8.35;CUST_39447_ PI430048170,CUST_73298_PI430048170,CUST_108340_PI430048170,CUST_118927_PI430048170,this four IncRNAs have not been annotation genes and have not found GeneSymbols, and by quantitative RT-PCR, which may be associated with osteoporosis patients' overall survival.

Conclusion: Analysis of this study revealed that dysregulated IncRNAs and mRNAs in osteoporosis patients and health people controls could affect the immune or metabolism system and musculoskeletal cell differentiation. The biological functions of those IncRNAs need to be further validated.

Keywords: IncRNA, Microarray profile, Musculoskeletal, Osteoporosis, Quantitative real-time PCR

\section{Background}

Osteoporosis (OP), is a systemic skeletal disorder, characterized by decreased bone mass, deterioration of the microarchitecture of bone tissue and increased fragility as well as consequent increase in risk of bone fracture,

\footnotetext{
* Correspondence: gzwl@gzucm.edu.cn

2Department of Orthopaedics, The Third Affiliated Hospital, Guangzhou University of Chinese Medicine, Guangzhou 510378, China

${ }^{3}$ Lingnan Medical Research Center of Guangzhou University of Chinese

Medicine, Guangzhou University of Chinese Medicine, Guangzhou 510405,

China

Full list of author information is available at the end of the article
}

which greatly affects people's life quality and even gives rise to the increased mortality, arousing extensive concerns among the population [1]. Osteopenia is less severe and refers to bone density that is below normal peak density but not low enough to be classified as osteoporosis [2].

Our understanding of bone-muscle crosstalk has been historically based on mechanical interactions between the bone and muscle. The bone is shaped by mechanical force applied by muscles, and the bone provides an attachment site for the muscle to maintain shape and

(c) The Author(s). 2019 Open Access This article is distributed under the terms of the Creative Commons Attribution 4.0 International License (http://creativecommons.org/licenses/by/4.0/), which permits unrestricted use, distribution, and 
drive locomotion [3]. The mechanical aspects of bone-muscle interactions are critical for normal development and movement and play a large role in changes of these tissues in disease and aging, yet the interactions between the bone and muscle are more complicated [4]. Just as our understanding of other organ system integrations has advanced, so too has our understanding of the complex endocrine-based crosstalk between the bone and muscle [3]. Bone and muscle anabolism are tightly coupled during growth and development. Conversely, bone and muscle catabolism occur during aging. Compromising either the bone or muscle by disease, disuse or aging affects both tissues but the cellular and molecular mechanisms linking these are not well understood [5]. Skeletal muscle and bone mass are influenced by various factors, including diet and nutrition, genetics, hormones, growth factors and mechanical stimuli [6]. The mass of bone and skeletal muscle is maintained in proportion to the mechanical loading experienced by the musculoskeletal system. For example, resistance training leads to an increase in muscle size and bone mineral density, which is associated with improved musculoskeletal fitness [7].

Long non-coding RNAs (lncRNAs) with length longer than 200 nucleotides are defined as transcripts that are not translated into protein [8]. Long non-coding RNA (lncRNA) regulates gene transcription and protein expressions genetically and epigenetically, and altered expressions result in immune or metabolism related desease development [9]. Among these newly discovered RNA elements, IncRNAs have been identified to have functional roles in a diverse range of cellular functions such as development, differentiation, cell fate, as well as disease pathogenesis [10]. Among the ncRNAs, two of them received lately extensive attention: miRNAs and lncRNAs. Several review the role of miRNAs and/or lncRNAs in adult skeletal myogenesis and muscle diseases [5]. LncRNAs can be divided into five broad categories: sense, antisense, bidirectional, intronic, and intergenic [11]. They are involved in diverse biological process and pathogenesis of disorders, such as osteoblast differentiation, osteosarcoma, and cardiovascular [12]. However, to our knowledge, little is known about lncRNAs expression profile in related to skeletal and muscle, and the potential pathways regulating osteoporosis remain poorly understood [13].

This pilot study aimed to identify aberrantly expressed lncRNAs and mRNAs profile of muscle disease and explore their potential functions in osteoporosis. In particular, using public databases, we identified the clinical significance of lncRNAs, it may be useful as diagnostic and prognostic biomarkers and provide novel therapeutic targets in related to skeletal and muscle diseases of the osteoporosis.

\section{Materials and methods}

Patients and tissue samples

A total of 3 pairs of osteoporosis and health patients skeletal muscle tissues were surgically obtained from adult patients undergoing treatment at The Third Affiliated Hospital, Guangzhou University of Chinese Medicine (Guangzhou,China) between January 2017 and March 2018. The detailed characteristics of the study subjects are summarizedin Table 1 . All the tissues were collected in anterior cruciate ligament reconstruction with autogenous tendon and fracture surgery patient. The fresh muscle were achieved from operating room and processed immediately in liquid nitrogen within 15 min and then storage in RNA Fixer Reagent (Bioteke,Beijing,China) at $-80^{\circ} \mathrm{C}$ prior to total RNA extraction. 3 pairs of tissues underwent microarray analysis and the remaining 10 tissues were used in validation studies by quantitative real-time polymerase chain reaction (qRT-PCR).

The Ethics Committee in Clinical Research of The Third Affiliated Hospital, Guangzhou University of Chinese Medical approved this study, and written informed consent was provided by all patients.

\section{Transcript analysis RNA extraction and purification}

Total RNA was extracted using TAKARA RNAiso Plus\#9109 following the manufacturer's instructions and checked for a RIN number to inspect RNA integrity by an Agilent Bioanalyzer 2100 (Agilent technologies, Santa Clara, CA, US). Qualified total RNA was further purified by RNeasy micro kit (Cat\#74004, QIAGEN, GmBH,

Table 1 Characteristics of the study subjects

\begin{tabular}{|c|c|c|c|c|c|}
\hline Number of tissues & Gender & Age (years) & Height $(\mathrm{cm})$ & Weight (kg) & Lumbar T-score \\
\hline NC-1 & Male & 33 & 176 & 78 & -1.2 \\
\hline NC-2 & Male & 30 & 167 & 63 & 1.4 \\
\hline NC-3 & Male & 60 & 170 & 66 & 1.4 \\
\hline OP-1 & Male & 43 & 178 & 80 & -3.4 \\
\hline OP-2 & Female & 52 & 156 & 58 & -3.7 \\
\hline OP-3 & Female & 68 & 152 & 46 & -2.8 \\
\hline
\end{tabular}


Germany) and RNase-Free DNase Set (Cat\#79254, QIAGEN, GmBH, Germany).

\section{RNA amplification and labeling}

Total RNA was amplified and labeled by Low Input Quick Amp Labeling Kit, One-Color (Cat.\#5190-2305, Agilent technologies, Santa Clara, CA, US), following the manufacturer's instructions. Labeled cRNA were purified by RNeasy mini kit (Cat.\# 74,106, QIAGEN, GmBH, Germany).

Each slide was hybridized with $1.65 \mu \mathrm{g}$ Cy3-labeled cRNA using Gene Expression Hybridization Kit (Cat.\# 5188-5242, Agilent technologies, Santa Clara, CA, US) in Hybridization Oven (Cat.\# G2545A, Agilent technologies, Santa Clara, CA, US), according to the manufacturer's instructions. After $17 \mathrm{~h}$ hybridization, slides were washed in staining dishes (Cat.\# 121, Thermo Shandon, Waltham, MA, US) with Gene Expression Wash Buffer Kit (Cat.\# 5188-5327, Agilent technologies, Santa Clara, CA, US), followed the manufacturer's instructions。 .

\section{Data acquisition}

Slides were scanned by Agilent Microarray Scanner (Cat\#G2565CA, Agilent technologies, Santa Clara, CA, US) with default settings, Dye channel: Green, Scan resolution $=3 \mu \mathrm{m}$, PMT 100\%, 20bit. Data were extracted with Feature Extraction software 10.7 (Agilent technologies, Santa Clara, CA, US). Raw data were normalized by Quantile algorithm, limma packages in R. We slect a standard threshold set for differentially expressed genes of a fold change $\geq 2.0$ and a value $\leq 0.05$.

\section{Gene ontology and Kyoto encyclopedia of genes and genomes pathway analyses}

Gene Ontology (GO) analysis was applied to analyze the main function of the differential expression genes according to the GO database. Pathway analysis was used to find out the significant pathway of the differential genes according to KEGG.

\section{IncRNA-mRNA coexpression networks}

$\mathrm{R}$ function cor.test (a test for association/correlation between paired sam-ples) was utilized to compute
Pearson's correlation coeficient to measure the gene coexpression. The lncRNAs (fold change $\geq 2.0$, value $\leq 0.01$ and length $0,10,000$ ), mRNAs (fold change $\geq 4.0$ and value $\leq 0.01$ ) were choosed to draw the network by Cytoscape.

According to these data, we built lncRNA-mRNA network using the correlation coefficients to examine interactions between IncRNA and mRNA. The value of "degree" in coexpression network indicated that one mRNA/lncRNA might be correlated with several lncRNAs/mRNAs.

\section{qRT-PCR analysis}

Total RNA was extracted and purified using standard methods (Life Technologies; RNA Easy, Qiagen, Valencia, CA,USA). Bestar qPCR RT Kit reverse transcription (Promega) was utilized to synthesize cDNA. 5 lncRNA (fold change $\geq 2.0$, value $\leq 0.01$ and length $0,3000 \mathrm{ChrX}$ ) expressions in sinonasal tissues were measured by qRT-PCR which was performed on the ABI7500 qPCR system with the primer pairs listed in Table 2. The raw quantifications were normalized to the beta-actin gene values for each sample and fold changes were shown as mean $\pm \mathrm{SD}$ in three independent experiments, each in triplicate.

\section{Statistical analysis}

All data were expressed as the mean \pm SD or proportions where appropriate. Expression levels skeletal muscle tissues from osteoporosis patients were analyzed by paired-sample -tests. values $<0.05$ (two-tailed) indicated statistical significance. The Statistical Program for Social Sciences (SPSS) 20.0 software was employed to perform all of the statistical analyses.

\section{Results}

Differentially expressed IncRNAs and mRNAs in osteoporosis

Volcano plots were used for assessing gene expression variation between osteoporosis and health patient groups. In total, 1594 lncRNAs displayed differential expression in osteoporosis, including 482 upregulated lncRNAs and 1112 down regulated lncRNAs. Of 886 mRNAs that showed differential expression, 436 were upregulated and 450 were downregulated. Among them,

Table 2 Real-time quantitative PCR primer sequences used in this study

\begin{tabular}{llll}
\hline Probe Name & Forward primers (5'-3') & Reverse primers (5'-3') & Amplicon size (bp) \\
\hline CUST_39447_PI430048170 & GTTCCCGAGGTGTCCGA & TCGTTAGTCGAGCTGAAACC & 148 \\
CUST_44695_PI430048170 & CGGTGAGGTAGAAGGAGAAC & TCTTCCTCAGCAGCACTTCC & 159 \\
CUST_73298_PI430048170 & AATCCTTGGCATTGTCTGTAA & AGCCAGTAGACGACACCAGC & 149 \\
CUST_108340_PI430048170 & GAACAAAGGAGCAACAGCCC & CCTGATAGGAATCTGCGAAAA & 83 \\
CUST_118927_PI430048170 & GAGGCTGGAATGCGGAGT & CACCTACTTTCCCTTGGCTATT & 160 \\
\hline
\end{tabular}


94 lncRNAs and 84 mRNAs were significantly upregulated, and 140 lncRNAs and 52 mRNAs were significantly downregulated $>2$-fold in osteoporosis. Hierarchical clustering analysis showed systematic variations in the expression of lncRNAs and mRNAs among samples. The data suggested that the expression of lncRNAs and mRNAs in osteoporosis differ from those in health people controls (Fig. 1). As expected, the IncRNA and mRNA expression proiles were distinguishing osteoporosis and normal tissue samples accurately based on the molecular signature.

Hierarchical clustering of the lncRNAs and mRNAs proile was performed using cluster 3.0.2; hierarchical clustering of the expression of the dysregulated lncRNAs and mRNAs based on centered Pearson correlation clearly separated osteoporosis tissues from corresponding normal tissues (Fig. 1). Out of the group of RNAs that were upregulated, lncRNA CUST_35058_PI430048170 and mRNA CUST_56753_PI430048170 showed the greatest degree of demonstrated upregulation, with 72.214 19842-and 36.48758725-fold increases, respectively; of those that were downregulated, IncRNA CUST_216 32_PI430048170 and mRNA CUST_139172_PI430048170 demonstrated the greatest degree of downregulation, with 15.62387857-and 18.19379213-fold decreases, respectively (Tables 3 and 4).

\section{Functional analysis of differentially expressed genes}

Until now, the functions of most lncRNAs have not been well annotated. Therefore, by analyzing differentially expressed mRNAs, we can forecast the role that lncRNAs play in osteoporosis. The GO and KEGG pathway analyses of differentially expressed lncRNAs and mRNAs could provide a clue about the osteoporosis

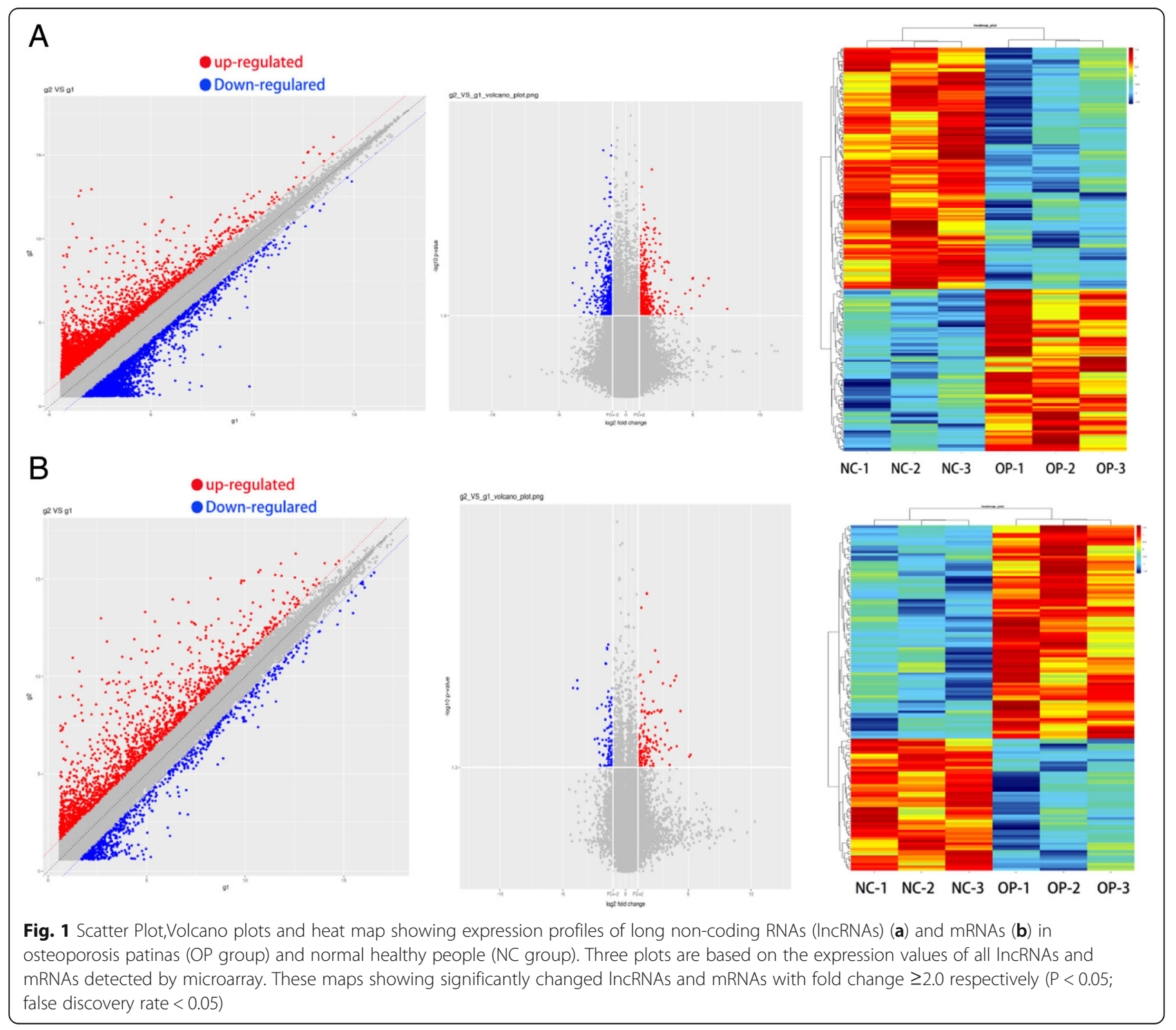


Table 3 Top 20 aberrantly expressed IncRNAs in microarray for 3 pairs of osteoporosis patients and health people skeletal muscle tissues

\begin{tabular}{|c|c|c|c|c|c|c|}
\hline ProbeName & $P$ & FC (abs) & Regulation & GeneSymbol & Accession & Chr \\
\hline CUST_35058_PI430048170 & 0.012556283 & 72.21419842 & up & MIR503HG & ENST00000440570 & $\operatorname{chr} x$ \\
\hline CUST_35059_PI430048170 & 0.016349269 & 55.40531579 & up & MIR503HG & NR_024607 & $\operatorname{chr} r$ \\
\hline CUST_126144_PI430048170 & 0.024118939 & 45.69384441 & up & MIR503HG & NR_024607 & $\operatorname{chrX}$ \\
\hline CUST_126145_PI430048170 & 0.012357324 & 32.40658055 & up & MIR503HG & ENST00000457876 & $\operatorname{chr} x$ \\
\hline CUST_35062_PI430048170 & 0.012723529 & 31.4726076 & up & MIR503HG & ENST00000457876 & $\operatorname{chr} x$ \\
\hline CUST_109345_PI430048170 & 0.038064776 & 29.70049534 & up & SAA3P & NR_026576 & chr11 \\
\hline CUST_41271_PI430048170 & 0.022023032 & 21.58734413 & up & RP11-283C24.1 & ENST00000578585 & chr17 \\
\hline CUST_21632_PI430048170 & 0.01486414 & 15.62387857 & down & VLDLR-AS1 & NR_015375 & chr9 \\
\hline CUST_110886_PI430048170 & 0.036123995 & 15.25447339 & up & RUNX1-IT1 & NR_026812 & chr21 \\
\hline CUST_98863_PI430048170 & 0.00857309 & 14.40142299 & down & - & Inc-RASA1-18:1 & chr5 \\
\hline CUST_80467_PI430048170 & 0.034308762 & 13.89512258 & down & VLDLR-AS1 & NR_015375 & chr9 \\
\hline CUST_56635_PI430048170 & 0.049061646 & 13.26382976 & up & - & Inc-PTEN-11:1 & chr10 \\
\hline CUST_112522_PI430048170 & 0.042135672 & 12.93582703 & up & RP11-283C24.1 & ENST00000578585 & chr17 \\
\hline CUST_10165_PI430048170 & 0.028081239 & 11.13188848 & down & VLDLR-AS1 & NR_015375 & chr9 \\
\hline CUST_71013_PI430048170 & 0.013220249 & 10.40135012 & up & - & Inc-INSM1-5:3 & chr20 \\
\hline CUST_113140_PI430048170 & 0.046258578 & 10.05254523 & up & - & Inc-CCDC144NL-4:1 & chr17 \\
\hline CUST_124876_PI430048170 & 0.0180434 & 9.786534613 & down & AC003090.1 & ENST00000446840 & chr7 \\
\hline CUST_21634_PI430048170 & 0.044021797 & 8.77824946 & down & VLDLR-AS1 & NR_015375 & chr9 \\
\hline CUST_71788_PI430048170 & 0.030034472 & 8.664571816 & up & - & Inc-RCAN1-6:1 & chr21 \\
\hline CUST_19829_PI430048170 & 0.022251937 & 8.525312141 & down & RP11-797H7.5 & ENST00000340779 & chr7 \\
\hline
\end{tabular}

disease process. We utilized all differentially expressed mRNAs for the GO analysis and found that the most enriched GO targeted by upregulated and downregulated transcripts were involved in anterior/posterior pattern specification, immune or metabolism system processes, and immune response (Fig. 2). In the KEGG pathway analysis, the down-and up-regulated mRNAs were found to be mostly enriched in muscle tissues, respectively (Fig. 3). Many genes involved in muscle tissues differentiation were dysregulated. A pathway network was constructed using 20 of the most significantly enriched pathways to illustrate the critical pathways in the process of osteoporosis. The bone metabolism pathway were considered to be the most central functions in the net because the exchanges with other pathways strongly depended on their existence (Fig. 3).

\section{Long non-coding RNA/mRNA coexpression network in osteoporosis}

We constructed the IncRNA-mRNA coexpression network to identify the interactions between mRNAs and lncRNAs. Transcriptome regulation involves a huge network, among which many transcripts form a complex web to function. Coexpression networks facilitate the intricate network based on gene screening methods that can be used to identify candidate biomarkers or therapeutic targets. The most crucial subnetwork was constructed by the transcripts with a high $k$-score, which would be the core regulatory modules of the entire coexpression network (Fig. 4). What is more, the transcripts in this network are widely distributed in all chromosomes, indicating the widely interconnected regulation network between lncRNAs and mRNAs (Fig. 4). This subnetwork includes four lncRNAs,lnc-CCT7-3:1,lnc-RASA118:1,NR_046609, and lnc-SEC23B-2:1, constituting probably the core of the network. The results implied that mRNA CDKN2B,CIDEC, and THBS4 may play key roles in osteoporosis process and development.

\section{qRT-PCR validation}

5 differentially expressed lncRNAs were randomly selected for validation by means of qRT-PCR according to the manufacturer's recommendations. CUST_108 340_PI430048170 was upregulated and CUST_39447_PI430048170,CUST_44695_PI430048170,CUST_73298_PI430048170,and CUST_118927_PI430048170 were downregulated in osteoporosis. The results of qRT-PCR were consistent with those of the microarray. All of the 5 lncRNAs were differentially expressed with the same trend (up-or downregulated) (Fig. 5). 
Table 4 Top 20 aberrantly expressed mRNAs in microarray for 3 pairs of osteoporosis patients and health people skeletal muscle tissues

\begin{tabular}{|c|c|c|c|c|c|c|}
\hline ProbeName & $P$ & FC (abs) & Regulation & GeneSymbol & Accession & Chr \\
\hline CUST_56753_PI430048170 & 0.0348289 & 36.48758725 & up & SCD & NM_005063 & chr10 \\
\hline CUST_133279_PI430048170 & 0.036740847 & 33.94274666 & up & SAA1 & NM_000331 & chr11 \\
\hline CUST_141790_PI430048170 & 0.009996764 & 20.86176002 & up & SAA4 & NM_006512 & chr11 \\
\hline CUST_139172_PI430048170 & 0.005145352 & 18.19379213 & down & SBK2 & NM_001101401 & chr19 \\
\hline CUST_141387_PI430048170 & 0.027240985 & 16.90436771 & up & SCD & NM_005063 & chr10 \\
\hline CUST_135342_PI430048170 & 0.003639094 & 16.28422029 & up & MRAP & NM_178817 & chr21 \\
\hline CUST_130964_PI430048170 & 0.004130947 & 14.9015582 & up & SAA4 & NM_006512 & chr11 \\
\hline CUST_138578_PI430048170 & 0.004133096 & 14.36030139 & down & AFP & NM_001134 & chr4 \\
\hline CUST_145430_PI430048170 & 0.005228882 & 14.17959829 & down & $\mathrm{PLCH} 1$ & NM_001130961 & chr3 \\
\hline CUST_132601_PI430048170 & 0.028566771 & 12.39092273 & up & DGAT2 & NM_032564 & chr11 \\
\hline CUST_128647_PI430048170 & 0.004831104 & 11.96333249 & up & PXDNL & NM_144651 & chr8 \\
\hline CUST_136078_PI430048170 & 0.046626016 & 11.41446117 & up & ANKRD1 & NM_014391 & chr10 \\
\hline CUST_139601_PI430048170 & 0.030211027 & 11.00284852 & up & ADIPOQ & NM_001177800 & chr3 \\
\hline CUST_142975_PI430048170 & 0.017278415 & 10.02050967 & up & PNPLA3 & NM_025225 & chr22 \\
\hline CUST_126930_PI430048170 & 0.014106518 & 8.930113104 & up & LGALS12 & NM_001142537 & chr11 \\
\hline CUST_142712_PI430048170 & 0.026107426 & 8.824194597 & up & RYR3 & NM_001036 & chr15 \\
\hline CUST_130312_PI430048170 & 0.004510607 & 7.885502819 & up & KLB & NM_175737 & chr4 \\
\hline CUST_52015_PI430048170 & 0.039281946 & 7.576008123 & up & RYR3 & NM_001036 & chr15 \\
\hline CUST_141047_PI430048170 & 0.009777851 & 7.231495622 & up & RBP4 & NM_006744 & chr10 \\
\hline CUST_136229_PI430048170 & 0.010210594 & 6.756306455 & up & TUSC5 & NM_172367 & chr17 \\
\hline
\end{tabular}

\section{Discussion}

It is well known that the mutation of genes and chromosomes contribute to the pathogenesis of leukemia. However, lncRNAs, the rising stars in biology, have just begun to be understood, and the majority of them have not yet been researched [14]. To provide some insights into the biological functions of lncRNAs in the pathogenesis of osteoporosis, we undertook a comprehensive analysis of lncRNA and mRNA profiling data from osteoporosis patients and health people, together with data from a public database. We identified the core lncRNAs and their functional annotations, and validated their expression by qRT-PCR. Overall, our work uncovered an interlaced transcripts network that is involved in osteoporosis development, in which lncRNAs play an indispensable role.

We explored the expression patterns of transcripts between osteoporosis patients and health people controls' muscleskeletal. Microarray data identified vast lncRNAs and mRNAs, supporting an extensive involvement of lncRNAs in osteoporosis. There were two important concepts that ran throughout our studies to handle the mass data. First, we simplified the complex transcript network by modularization. The GO and KEGG pathway analyses divided mRNAs into several functional modules, which are related to immunity, metabolism, osteoclast differentiation, hematopoiesis, and TGF-beta signaling pathway, indicating the validity of the microarray [15]. Cytoscape was used to construct co-expressed networks from the public and our microarray data, respectively, and then the networks were facilitated into several sub-networks through the k-score method. In the same way, lncRNAs were attributed their correlated functional mRNA modules through the co-expressed network and GO annotations. Overall, modularization contributes to simplifying the intricate network into modules, which were like "big genes". Further analysis of public data showed that the IncRNA may regulate osteoclast differentiation, leading to the decrease bone mass, cause to osteoporosis happened [16]. Finally, five lncRNAs, CUST_39447_PI430048170,CUST_44695_PI430048170,CUST_73298_PI430048170,CUST_108340_PI430048170,and

CUST_118927_PI430048170 were confirmed as significantly differentially expressed in osteoporosis patients and healthy controls by qRT-PCR.

Our work clearly indicated an important role for lncRNAs in OP. However, many lncRNAs were excluded as they failed to be allocated to functional modules and have not been included in public data. It was difficult to originally understand the functions and targets of these lncRNAs, which may also play a key role in osteoporosis 


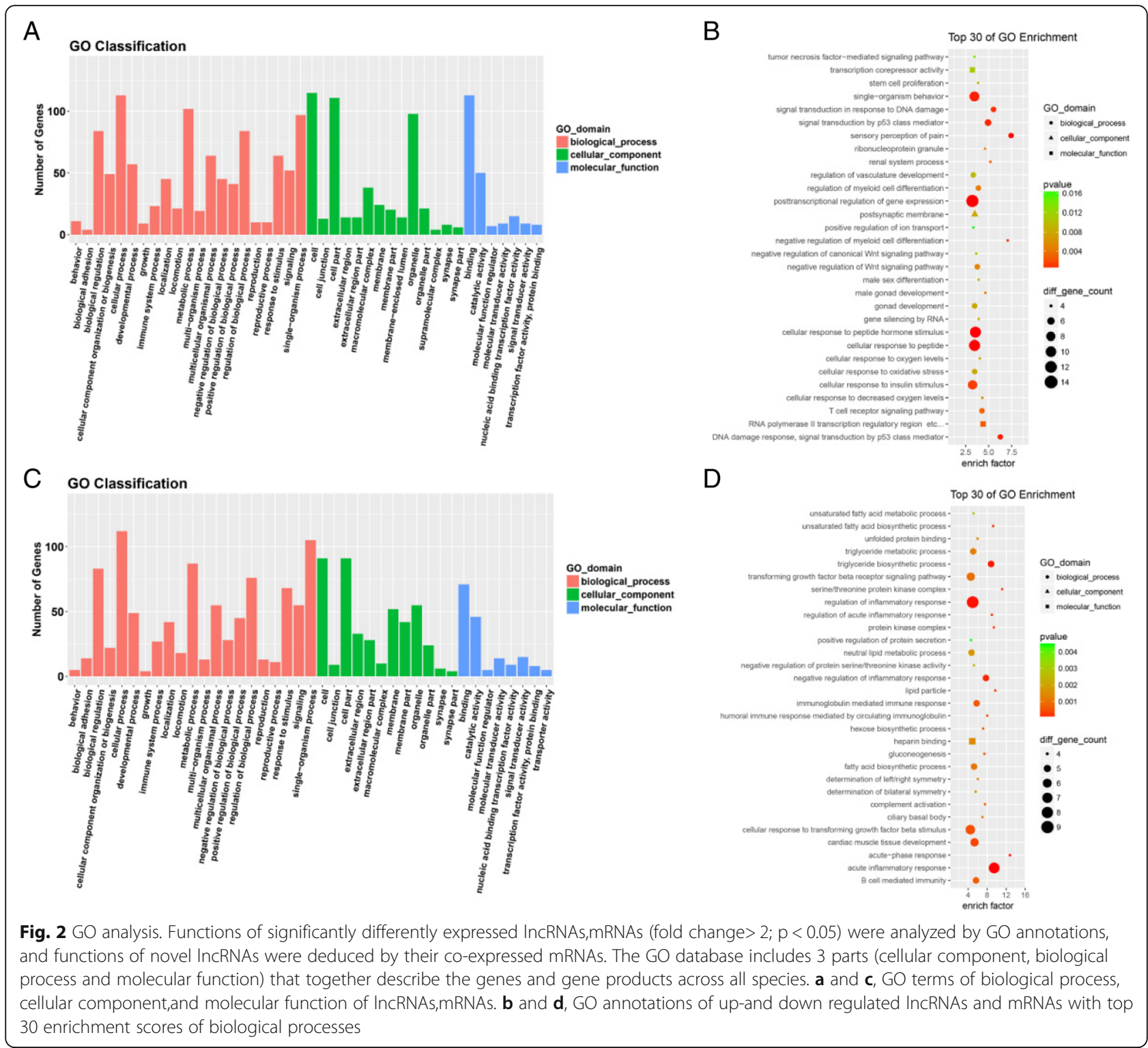

[17]. In addition, our analysis showed that IncRNA lnc-CCT7-3:1 was highly correlated with osteoporosis, its neighboring gene. The lncRNA may be directly or indirectly correlated with osteoporosis and there may be additional transcripts involved in the IncRNA-associated biological process [18]. The lncRNA's biological functions need to be validated further.

The last decades witnessed the discovery of biological functions for non-coding RNA, which triggered the recognition that RNA is not only a simple hinge of the central dogma but also directly takes part in the regulation of biological networks [19]. With the development of next-generation sequencing, especially in terms of depth and scale, significant data has been accumulated. We have to recognize the system is so complex that it is beyond the initial recognition. Fortunately, the progress of methodology simplifies the networks. Through modularization, thousands of transcripts can be facilitated into several "big genes," and then the core lncRNAs of each module can be researched in details. Moreover, the active application of accumulated public data will help us to make the functions of lncRNAs more clear. Hopefully, this study can provide a reference for the broad analysis of OP data.

Skeletal muscle possesses a remarkable ability to adapt to various physiologic conditions [20]. AMPK is a sensor of intracellular energy status that maintains energy stores by fine-tuning anabolic and catabolic pathways [21]. At the same time, skeletal muscle has been shown to be important for regulating whole-body metabolism. 


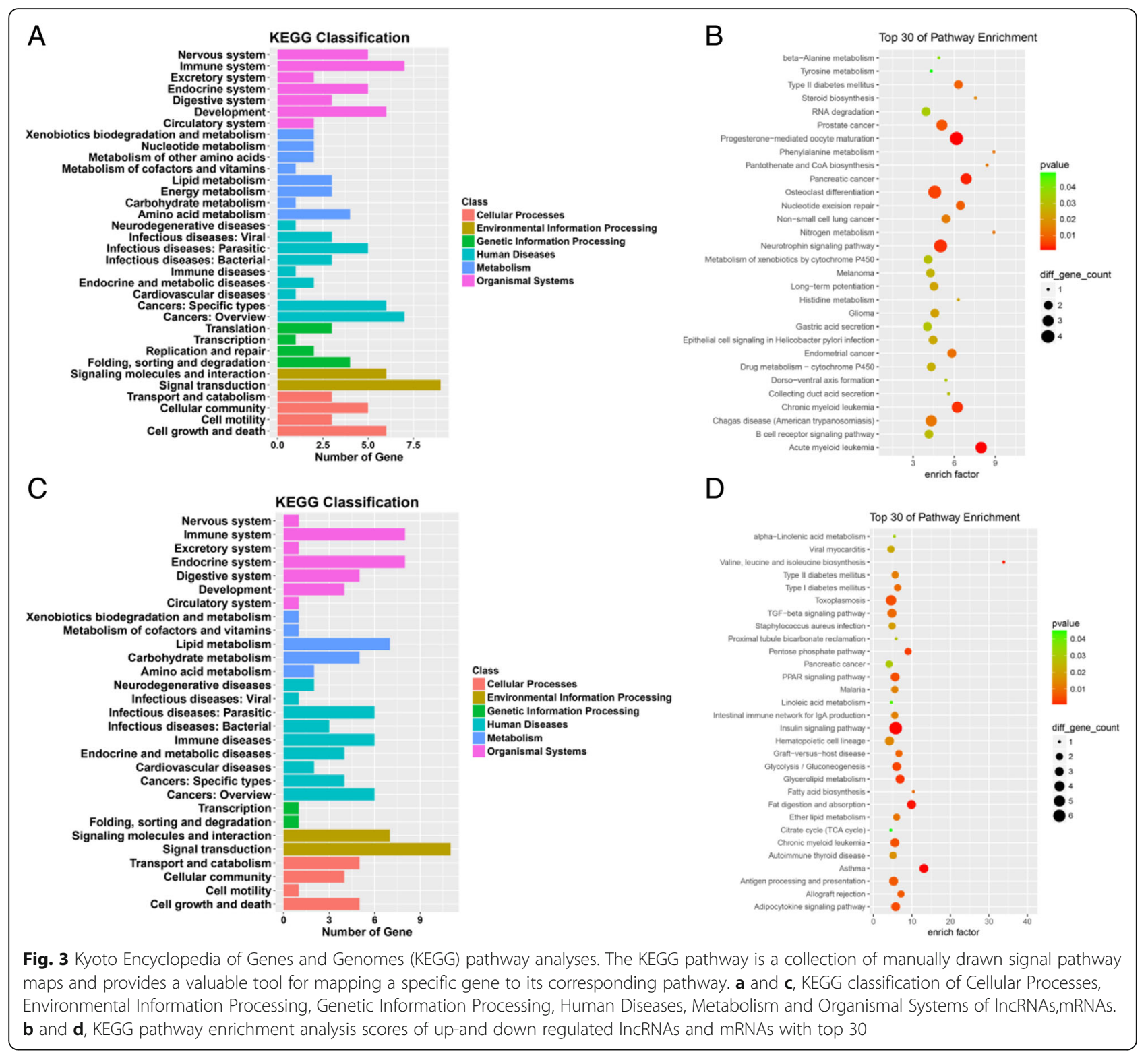

Skeletal muscle demonstrates high malleability and can adapt its contractile composition and metabolic properties in response to a number of physiologic conditions [22]. However, bone mass and skeletal muscle mass are controlled by factors such as genetics, diet and nutrition, growth factors and mechanical stimuli. Whereas increased mechanical loading of the musculoskeletal system stimulates an increase in the mass and strength of skeletal muscle and bone, reduced mechanical loading and disuse rapidly promote a decreasein musculoskeletal mass, strength and ultimately performance [26]. Moreover, skeletal muscle atrophy often occursinparallel tobonelossand activity; musclemassand strength influence bone mass [23]. However, because the mechanisms that regulate mechanotransduction in bone and muscle are complex, it is not entirely clear which specific mechanisms operate in synergy during musculoskeletal disuse [24]. Treatment options for muscle disuse atrophy and osteoporosis are currently limited, with rehabilitative strength training proving to be the most effective method to restore musculoskeletal mass; however, long periods of rehabilitation are necessary to restore muscle, bone and locomotor performance following artificial immobilisation in non-hibernating mammals [25]. Muscules and bones are the main part of the motor system, regulating processes such as endocrine and metabolism [26]. IGF-1 in muscle tissue promotes bone development by improving muscle mass and strength, and also 


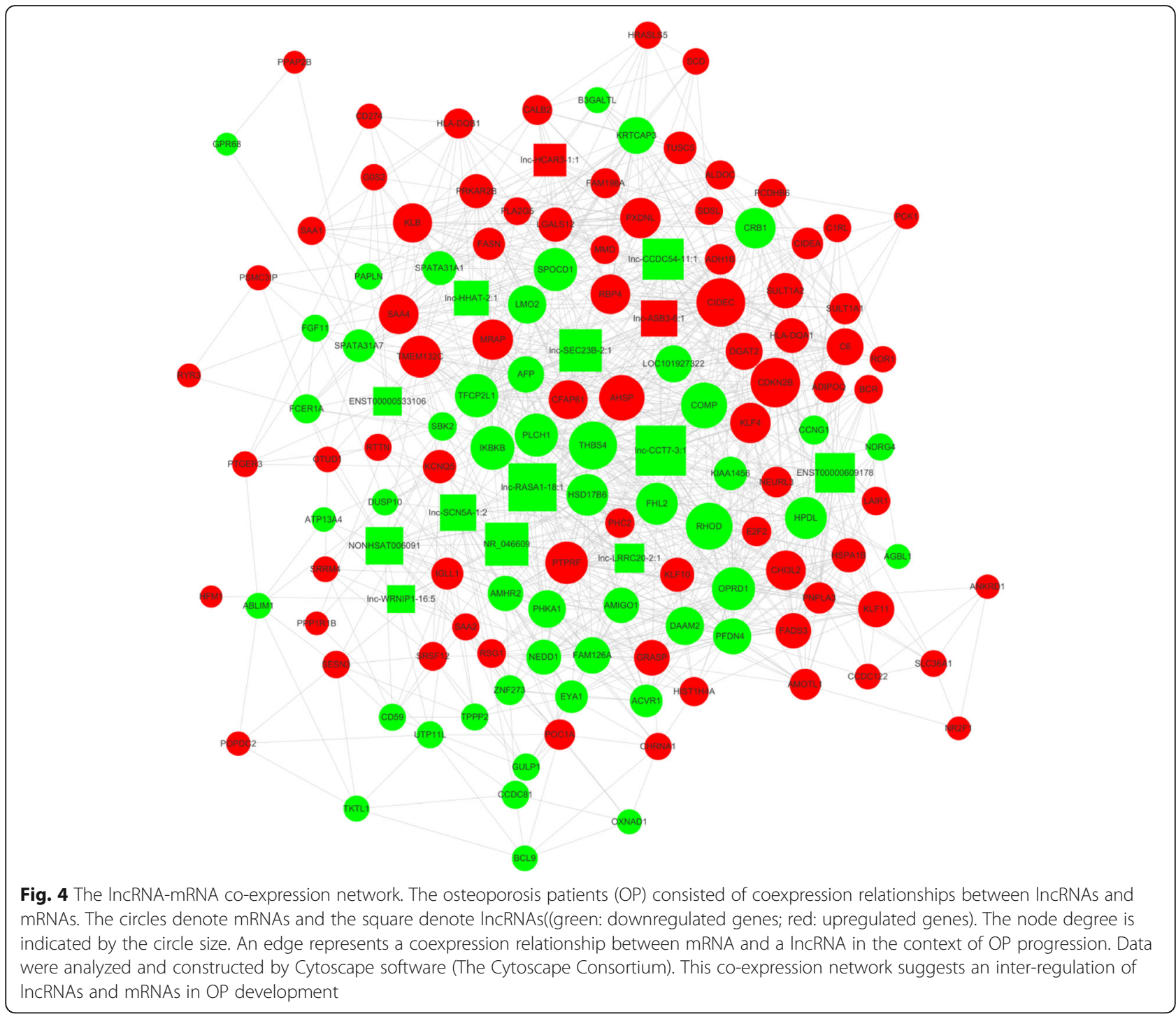

directly promotes bone development, increasing bone mineral content and bone density [27]. Myostatin is most abundantly expressed in skeletal muscle tissue, inhibits muscle growth and regeneration, and promotes osteoclast differentiation, inhibiting TNF- $\alpha$-induced osteoclast differentiation [28]. Indian Hedgehog (Ihh), a signaling molecule secreted by chondrocytes and osteoblasts, promotes muscle growth. Ihh in bone can directly promote muscle growth [29]. Osteocalcin (Glu-OC) partially repairs muscles with impaired function. The gap junction protein Connexin 43 in bone cells directly regulates muscle growth and function [30]. The close connection between bone and muscle is not only related to the mechanics of its interaction, but also to the complex and precise endocrine regulation and some common molecular signaling pathways [31]. The new recent study that significant differences in the expression of lncRNAs,
mRNAs, circRNAs, and miRNAs between postmenopausal OP patients and healthy controls, and the functions of the RNAs were also identified based on our RNA-seq analysis [32]. Taken together, our study indicated that lncRNAs and mRNA could associate with the occurrence of OP and may be as possible biomarkers and target genes in OP.

\section{Conclusions}

Insummary, to our knowledge, our study analysis of lncRNA expression profile in osteoporosis. The results show that genes regulated by these lncRNAs are involved in TGF-beta signaling pathways as a proof of principle, or influence osteoclast differentiation. This may offer new insights into pathogenesis and could be a promising way to dissect the molecular pathogenesis of this refractory bone metabolism desease. Our study lays 


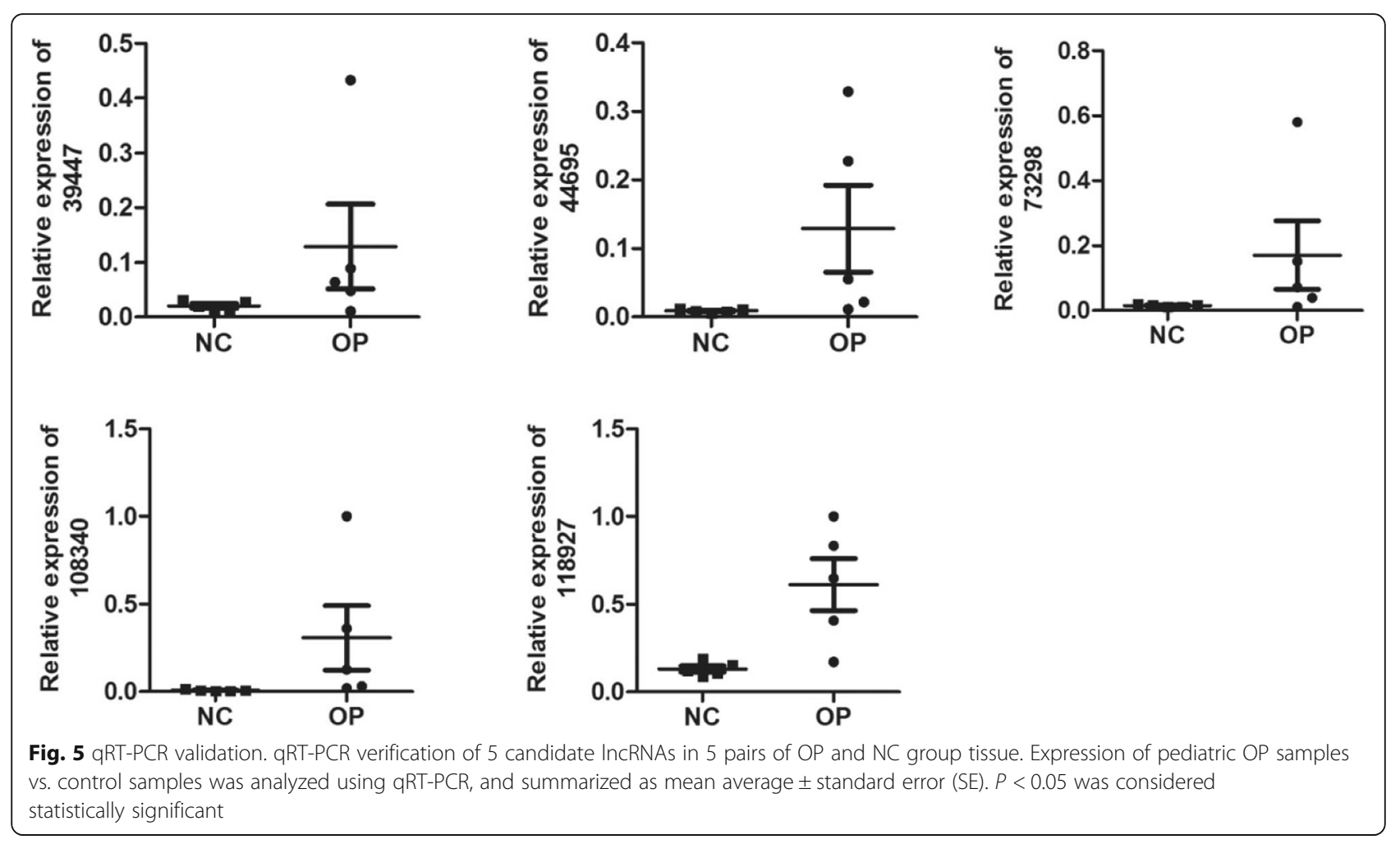

the foundation for further investigation of this disease. Further large scale studies are warranted to provide convincing evidence for clarifying the functions of lncRNAs in osteoporosis and determining whether these lncRNAs can serve as new diagnostic biomarkers, prognostic factors for survival, and therapeutic targets in osteoporosis.

\section{Abbreviations}

GO: Gene Ontology; KEGG: Kyoto Encyclopedia of Genes and Genomes; LnCRNA: Long non-coding RNA; OP: Osteoporosis; GRT-PCR: Quantitative polymerase chain reaction

\section{Acknowledgements}

Not applicable.

\section{Funding}

This study was supported by Grants from the National Natural Science Foundation of China (81674004;81673786).

\section{Availability of data and materials}

All data generated or analysed during this study are included in this published article.

\section{Authors' contributions}

LW and HH conceived and designed the experiments; SL, SC and JH performed the experiments; HW and SL performed data analysis; SL and HW contributed to sample collection; SL wrote the paper; LW assisted with writing and proofreading. All authors read and approved the final manuscript.

\section{Ethics approval and consent to participate}

This study was approved by the Internal Review and the Ethics Boards of Guangzhou University of Chinese Medicine and The Third Affiliated Hospital, Guangzhou University of Chinese Medicine. Informed written consent was obtained from all study subjects.
Consent for publication

Not applicable.

\section{Competing interests}

The authors declare that they have no competing interests.

\section{Publisher's Note}

Springer Nature remains neutral with regard to jurisdictional claims in published maps and institutional affiliations.

\section{Author details \\ ${ }^{1}$ Guangzhou University of Chinese Medicine, Guangzhou 510405, China. 2Department of Orthopaedics, The Third Affiliated Hospital, Guangzhou University of Chinese Medicine, Guangzhou 510378, China. ${ }^{3}$ Lingnan Medical Research Center of Guangzhou University of Chinese Medicine, Guangzhou University of Chinese Medicine, Guangzhou 510405, China.}

Received: 31 January 2019 Accepted: 15 May 2019

Published online: 31 May 2019

\section{References}

1. Cauley JA. Osteoporosis: fracture epidemiology update 2016. Curr Opin Rheumatol. 2017;29:150-6.

2. Tong X, Gu P-c, Xu S-Z, Lin X-j. Long non-coding RNA-DANCR in human circulating monocytes: a potential biomarker associated with postmenopausal osteoporosis. Biosci Biotechnol Biochem. 2015;79(5):732-7.

3. Picca $A$, Calvani $R$, et al. Bone-muscle crosstalk: unraveling new therapeutic targets for osteoporosis. Curr Pharm Des. 2017;23:1-8.

4. Ferrucci $\mathrm{L}$, Baroni M, Ranchelli A, et al. Interaction between bone and muscle in older persons with mobility limitations. Curr Pharm Des. 2014;20: 3178-97.

5. Goodman CA, Hornberger TA, Robling AG. Bone and skeletal muscle: key players in mechanotransduction and potential overlapping mechanisms. Bone. 2015;80:24-36

6. Reilly BD, Franklin CE. Prevention of muscle wasting and osteoporosis: the value of examining novel animal models. J Exp Biol. 2016;219:2582-95. 
7. Brooks NE, Myburgh KH. Skeletal muscle wasting with disuse atrophy is multi-dimensional: the response and interaction of myonuclei, satellite cells and signaling pathways. Front Physiol. 2014;5:99.

8. Cao J. The functional role of long non-coding RNAs and epigenetics. Biol Proced Online. 2014;16:11.

9. Li G, Zhang H, Wan X, Yang X, Zhu C, Wang A, He L, Miao R, Chen S and Zhao H: Long noncoding RNA plays a key role in metastasis and prognosis of hepatocellular carcinoma. Biomed Res Int 2014: 780521, 2014.

10. McMullen JR, Drew BG. Long non-coding RNAs (IncRNAs) in skeletal and cardiac muscle: potential therapeutic and diagnostic targets? Clin Sci. 2016; 130:2245-56.

11. Kashi K, Henderson L, Bonetti A, Carninci P. Discovery and functional analysis of IncRNAs: methodologies to investigate an uncharacterized transcriptome. Biochim Biophys Acta. 2016;1859:3-15.

12. Beermann J, Piccoli MT, Viereck J, Thum T. Non-coding RNAs in development and disease: background, mechanisms, and therapeutic approaches. Physiol Rev. 2016;96:1297-325.

13. Nelson BR, Makarewich CA, Anderson DM, Winders BR, Troupes CD, Wu F, Reese AL, McAnally JR, Chen X, Kavalali ET, et al. A peptide encoded by a transcript annotated as long noncoding RNA enhances SERCA activity in muscle. Science. 2016;351:271-5.

14. Tong X, Gu PC, Xu SZ, Lin XJ. Long non-coding RNA-DANCR in human circulating monocytes:a potential biomarker associated with postmenopausal osteoporosis. Biosci Biotechnol Biochem. 2015;79:732-7.

15. Mao X, Cai T, Olyarchuk JG, Wei L. Automated genome annotation and pathway identification using the KEGG Orthology (KO) as a controlled vocabulary. Bioinformatics. 2005;21:3787-93.

16. Jin C, Zheng Y, Huang Y, Liu Y, Jia L, Zhou Y. Long non-coding RNA MIAT knockdown promotes osteogenic differentiation of human adipose-derived stem cells. Cell Biol Int. 2017;41:33-41.

17. Gao KT, Lian D. Long non-coding RNA MALAT1is an independent prognostic factor of osteosarcoma. Eur Rev Med Pharmacol Sci. 2016;20:3561-5.

18. Bhan A, Mandal SS. Long noncoding RNAs: emerging stars in gene regulation, epigenetics and human disease. ChemMed-Chem. 2014;9:1932-56.

19. Prabhakar B, Zhong XB, Rasmussen TP. Exploiting long noncoding RNAs as pharmacological targets to modulate epigenetic diseases. Yale J Biol Med. 2017;90:73-86

20. Egan B, Zierath JR. Exercise metabolism and the molecular regulation of skeletal muscle adaptation. Cell Metab. 2013:17:162-84.

21. Gowans GJ, Hardie DG. AMPK: a cellular energy sensor primarily regulated by AMP. Biochem Soc Trans. 2014;42:71-5.

22. Anderson DM, et al. A micropeptide encoded by a putative long noncoding RNA regulates muscle performance. Cell. 2015;160:595-606.

23. Swift JM, Nilsson Ml, Hogan HA, Sumner LR, Bloomfield SA. Simulated resistance training during hindlimb unloading abolishes disuse bone loss and maintains muscle strength. J Bone Miner Res. 2010;25:564-74.

24. Goodman CA. The role of mTORC1 in regulating protein synthesis and skeletal muscle mass in response to various mechanical stimuli. Rev Physiol Biochem Pharmacol. 2014;166:43-95.

25. Cotton CJ. Skeletal muscle mass and composition during mammalian hibernation. J Exp Biol. 2016;219:226-34.

26. Dallas SL, Prideaux M, Bonewald LF. The osteocyte:an endocrine cell and more. Endocr Rev. 2013;34:658-90.

27. Banu J, Wang L, Kalu DN. Effects of increased muscle mass on bone in male mice overexpressing IGFI in skeletal muscles. In: Calcif tissue Int, 2008, 73. p. 196-201.

28. Dankbar B, Fennen M, Brunert D, et al. Myostatin is a direct regulator of osteoclast differentiation and its inhibition reduces inflammatory joint destruction in mice. Nat Med. 2015;21:1085-90.

29. Bren-Mattison Y, Hausburg M, Olwin BB. Growth of limb muscle is dependent on skeletal-derived Indian hedgehog. Dev Biol. 2011;356:486-95.

30. Shen H, Grimston S, Civitelli R, et al. Deletion of connexin43 in osteoblasts /osteocytes leads to impaired muscle formation in mice. J Bone Miner Res. 2015;30:596-605.

31. Borsheim E, Herndon DN, Hawkins HK, et al. Pamidronate attenuates muscle loss after pediatric burn injury. J Bone Miner Res. 2014;29:1369-72.

32. Jin $D$, Wu X, Yu H, et al. Systematic analysis of IncRNAs, mRNAs, circRNAs and miRNAs in patients with postmenopausal osteoporosis. Am J Transl Res. 2018;10(5):1498-510

\section{Ready to submit your research? Choose BMC and benefit from:}

- fast, convenient online submission

- thorough peer review by experienced researchers in your field

- rapid publication on acceptance

- support for research data, including large and complex data types

- gold Open Access which fosters wider collaboration and increased citations

- maximum visibility for your research: over $100 \mathrm{M}$ website views per year

At BMC, research is always in progress.

Learn more biomedcentral.com/submissions 